\title{
A FAMILY OF LIE ALGEBRAS NOT EXTENDIBLE TO A FAMILY OF LIE GROUPS
}

\author{
DON COPPERSMITH
}

\begin{abstract}
We produce an analytic family of finite-dimensional Lie algebras, parameterized by a smooth Hausdorff manifold, which does not correspond to the Lie algebra of any Hausdorff (separable) family of Lie groups. This answers a question of Douady and Lazard.
\end{abstract}

Douady and Lazard [1] define an analytic family of Lie groups and an analytic family of Lie algebras. They prove an analogue of Lie's Third Theorem, namely, if $\mathfrak{h}$ is an analytic family of Lie algebras parameterized by a finite-dimensional analytic manifold $S$, then there is a nonseparable (i.e. not necessarily separable) family $\mathfrak{g}$ of Lie groups, parameterized by $S$, whose Lie algebra corresponds to $\mathfrak{h}$. They ask whether an analogous theorem still holds locally (around each point of $S$ ) if one requires $\mathfrak{G}$ to be separable. We give here an example which provides a negative answer.

Definition. An analytic family of Lie algebras parameterized by $S$ is a (separable) fiber space $\mathfrak{h}$ over a finite-dimensional analytic manifold $S$, whose fiber $h_{s}$ at each point $s \in S$ is a finite-dimensional vector space, together with an analytic antisymmetric bilinear fiber map $[]:, \mathfrak{h} \times{ }_{s} \mathfrak{h} \rightarrow \mathfrak{h}$ giving each fiber $h_{s}$ the structure of a Lie algebra.

DEFINITION. A nonseparable analytic family of Lie groups parameterized by $S$ is a nonseparable (i.e. not necessarily separable) analytic manifold $\mathfrak{B}$ together with a surjective submersion $\pi: \mathfrak{S} \rightarrow S$, a section $e: S \rightarrow \mathfrak{B}$, and a morphism $M: \mathfrak{S} \times s \mathfrak{G} \rightarrow \mathfrak{G}$, such that for each $s \in S$, the fiber $\pi^{-1}(s)=H_{s}$ has a Lie group structure given by multiplication $M \mid H_{s} \times H_{s}$ and identity $e(s)$.

REMARK. The base space $S$ and fibers $H_{s}$ are all separable manifolds; only the total space $\mathfrak{S}$ is allowed to be nonseparable (non-Hausdorff).

DEFINITION. A separable analytic family of Lie groups parameterized by $S$ is a nonseparable family for which the total space $\mathfrak{B}$ is separable.

Example. Let $S=\mathbf{R}^{2}=\{(t, u) \mid t, u \in \mathbf{R}\}$. Let $\mathfrak{h}$ be the family of fourdimensional real Lie algebras parameterized by $S$, whose fiber $h_{s}$ over $s=(t, u)$ has basis $\left\{x_{s}, y_{s}, z_{s}, w_{s}\right\}$ and bracket given by

$$
\begin{gathered}
{\left[w_{s}, x_{s}\right]=\left[w_{s}, y_{s}\right]=\left[w_{s}, z_{s}\right]=0,} \\
{\left[x_{s}, z_{s}\right]=-y_{s}, \quad\left[y_{s}, z_{s}\right]=x_{s}, \quad\left[x_{s}, y_{s}\right]=t z_{s}+u w_{s} .}
\end{gathered}
$$

Received by the editors November 22, 1976 and, in revised form, June 24, 1977.

AMS (MOS) subject classifications (1970). Primary 22E15. 
The Jacobi identity is satisfied for each $s$.

Suppose we have a neighborhood $S^{\prime}$ of 0 in $S$ and a separable family $\mathscr{S}$ of Lie groups parameterized by $S^{\prime}$, whose Lie algebras correspond to $\mathfrak{h} \mid S^{\prime}$. Let $s=(t, u) \in S^{\prime}, t>0, u / t=r$. In $h_{s}$, the elements $x_{s} / \sqrt{t}, y_{s} / \sqrt{t}, z_{s}+r w_{s}$ span a subalgebra isomorphic to $s o(3)$ and satisfy the usual bracket relations. In the Lie group $H_{s}$ over $s$, since the subgroup generated by this subalgebra can only be $\mathrm{SO}(3)$ or its double cover, we must have exp $4 \pi\left(z_{s}+r w_{s}\right)=e(s)$ (the identity in $H_{s}$ ). Fixing $r$, this equation holds for each $s \in S^{\prime}$ on the ray $u=r t, t>0$, and, by the assumption that $\mathscr{S}$ is separable, it holds at the fiber over 0 as well: $\exp 4 \pi\left(z_{0}+r w_{0}\right)=e(0)$. Since $\left[z_{0}, w_{0}\right]=0$, this implies $\left(\exp 4 \pi z_{0}\right)\left(\exp 4 \pi r w_{0}\right)=e(0)$. This holds for all values of $r$. Differentiating with respect to $r$ we find $w_{0}=0$, a contradiction.

Thus no separable family of Lie groups has $\mathfrak{h}$ as its Lie algebra.

REMARK. This failure of the extendibility of Lie's Third Theorem to separable analytic families of Lie algebras and Lie groups suggests that the proper category in which to work is that of nonseparable families of Lie groups. This suggestion is strengthened, for example, by the fact that in this nonseparable family, one is able to prove [2] the standard results from deformation theory (rigidity and universal deformation theorems), while in the separable category the expected universal deformation theorem fails.

(Roughly, the universal deformation theorem says that if $H$ is a Lie group with $H^{3}(H, h)=0$ (Lie group cohomology [3]), then there is a nonseparable family of Lie groups, whose fiber over 0 is equal to $H$, and whose "infinitesimal deformation" at 0 is all of $H^{2}(H, h)$. The analogous statement for separable families fails, as may be seen by letting $H$ be the 3-fold cover of $E(2)$ (the group of rigid motions of $\mathbf{R}^{2}$ ). We have $H^{3}(H, h)=0$, but if a separable universal deformation of $H$ existed, a fiber near 0 would have to be a connected 3-fold cover of $\mathrm{SO}(3)$, which does not exist.)

These results are amplified in [2].

\section{REFERENCES}

1. A. Douady and M. Lazard, Espaces fibrés en algèbres de Lie et en groupes, Invent. Math. 1 (1966), 133-151.

2. D. Coppersmith, Deformations of Lie groups and Lie algebras, Ph. D. Dissertation, Harvard Univ., 1977 (unpublished).

3. G. Hochschild and G. D. Mostow, Cohomology of Lie groups, Illinois J. Math. 6 (1962), $367-401$.

IBM T. J. Watson Research Center, Box 218, Yorktown Heights, New YorK 10598 